\title{
Decision making in Resection of Oral Cancer: How to go about it
}

\author{
Garima Rawat ${ }^{1 *}$ and Anshuman Kumar ${ }^{2}$ \\ ${ }^{1}$ Department of Pathology, Dharamshila Narayana Superspeciality Hospital, India \\ ${ }^{2}$ Director-Surgical Oncology, Dharamshila Narayana Superspeciality Hospital, India
}

Submission: August 12, 2020; Published: September 23, 2020

*Corresponding author: Garima Rawat, Senior Resident, Department of Pathology, Dharamshila Narayana Superspeciality Hospital, New Delhi, India

\section{Abstract}

Oral cancer is a global health problem with growing incidence and mortality rates. As highlighted by International Agency for Research on Cancer and World Health Organization on 2014, the early diagnosis of oral cancer reduces morbidity and mortality rates. The most accepted mode of initial definitive treatment for most oral cancers is surgical management. Various factors related to the primary Tumour and the patient affect choice of treatment. Primary site, location, size, proximity to bone, and depth of infiltration are factors which influence a particular surgical approach. Tumour' s that approach or involve the mandible require specific understanding of the mechanism of bone involvement. Over time the role of surgery in primary squamous cell carcinomas in other sites in the head and neck has evolved with integration of multidisciplinary treatment approaches employing chemotherapy and radiotherapy either sequentially or concurrently. In this article, we throw light on the numerous component that influence the decision making of the surgeon before surgical resection in oral cancer.

Keywords: Oral cancer; Multidisciplinary approach; Surgical resection

\section{Introduction}

Oral cancer is the most common malignant disorder of the oral cavity, accounting for over $90 \%$ of all malignant neoplasms in this region. It accounts for majority of morbidity and mortality in individuals affected by head and neck cancer. They include a group of neoplasms affecting any region of the oral cavity, pharyngeal region, and salivary glands. It accounts for $2 \%-4 \%$ of all cancers worldwide and is ranked as the sixth leading cause of cancer mortality globally. About $30 \%-80 \%$ of the oral malignancies arise from premalignant disorders. Oral cancer has a multifactorial etiology. The various factors implicated in the etiopathogenesis of OSCC include smoking, chewing betel quid/tobacco, and alcohol intake separately or synergistically, viruses, genetic factors, environmental factors, and gene environment interactions $[1,2]$.

For oral cancer, the treatment modalities can be classified according to the stage of the disease-

a. Stage I \& II - Surgery

b. Stage II, III, IVA- Multimodality- including surgery along with adjuvant radiation therapy and/ or chemotherapy

c. Stage IVB \&C- Beyond the scope of surgical intervention and only Radiation therapy or chemotherapy can be used [3].

Surgery is the most well-established mode of initial definitive treatment for most oral cancers, with the longest history of being the most accepted method of treatment for years over other treatment modalities. With the introduction of ionizing radiation, upon discovery of radium, radiation therapy (RT) became an important means of nonsurgical treatment of oral carcinoma. Although, in majority of patients with advanced stage of oral cancer, radiotherapy (RT) is administered as an adjuvant treatment modality in conjunction with suger. Chemotherapy in the management of oral carcinoma was considered palliative during the early years, though with the introduction of Cisplatinum, clinical trials of induction chemotherapy demonstrated that response to chemotherapy was observed in a significant number of patients. However, unlike other sites in the head and neck area, the response to induction chemotherapy did not translate into long term control of primary oral squamous cell carcinomas. Targeted therapies with EGFR inhibitors are an active area of investigation currently. Immunotherapy and gene therapy are also areas of research where further work needs to be done $[2,3]$.

\section{Factors Affecting Choice of Treatment}

The factors that affect the choice of initial treatment are those related to the characteristics of the primary tumor, those related to the patient and those related to the treatment team. Thus, they are categorized as follows 


\section{Global Journal of Otolaryngology}
a. Tumour factors
b. Patient factors
c. Physician factors.

While deciding the optimal therapy for patients with oral cancer, one should consider these three sets of parameters in initial treatment planning. As the goal of treatment of cancer of the oral cavity is to eradicate the cancer, preserve or restore form and function, minimize the sequelae of treatment, and prevent any subsequent new primary cancers. To achieve these aims and objectives, the available treatment modalities include surgery, radiotherapy, chemotherapy, combined modality treatments and primary and secondary prevention strategies including lifestyle changes as well as chemoprevention.

\section{Tumour factors}

The tumour factors that affect the choice of initial treatment of oral cancer are primary site, size (T Stage), location (anterior versus posterior), proximity to bone (mandible or maxilla), status of cervical lymph nodes, previous treatment, and histology (type, grade and depth of invasion) [4]. The biological behaviour of primary cancers in the oral cavity is dependent upon the location of the disease, for example, cancers involving the hard palate and upper gingiva behave relatively indolently and lesser chances of regional lymph node metastases. Whereas, cancers involving the tongue, floor of the mouth have a high risk of regional lymph node metastases with an adverse impact on prognosis. The size of the primary tumour directly impacts the decision regarding choice of initial treatment. Small superficial primary tumours are easily accessible for surgical resection, while larger tumours require more extensive surgical approaches for exposure and excision. Also, larger size predisposes to higher risk of regional lymph node metastases mandating adjuvant therapy.

Neck dissection is an integral part of surgery in case of clinically palpable neck nodes although, the extent of level of cervical lymph nodes dissected will be dependent on the extent of nodal metastases and the location of palpable lymph nodes. The patterns of regional lymph node metastases from primary cancers of the oral cavity are well established and sequential progression of metastatic spread occurs from primary oral cancers [5]. The sentinel lymph nodes for oral cancer are located at levels 1,2 , and 3 in the neck with a relatively infrequent dissemination to level 4 . Skip metastases to level 5 does not occur. Therefore, in planning elective dissection of regional lymph nodes for micro metastases, clearance of lymph nodes at level 5 is not necessary. On the other hand, when gross metastases are present in the anterior triangle of the neck, a comprehensive dissection of all five levels of lymph nodes is recommended $[6,7]$.

The histology of the primary tumour is an important parameter which influences selection of initial treatment. Squamous carcinomas greatly predominate in the oral cavity and account for over ninety percent of all primary malignant neoplasms in the oral cavity. Patients with advanced stage disease, that is those presenting with spread to regional lymph nodes or with large primary tumours (T3 and T4), are candidates for consideration of combined modality treatment.

\section{Patient factors}

Many factors relative to patient characteristics play an important role in the selection of initial treatment for oral cancer. These include patient's age, general medical condition, and tolerance of treatment, occupation of the patient, acceptance and compliance by the patient, lifestyle (smoking and drinking) and other socioeconomic considerations. The ability of the patient to tolerate an optimal therapeutic program is equally important parameter which influences the choice of initial treatment. The patient's acceptance of and compliance with the proposed treatment also play a crucial role in designing an optimal treatment program for the tumour $[3,7]$.

\section{Physician factors}

The factors related to the treatment delivery team are also important in making the selection of initial definitive treatment for oral cancer. Expertise in various disciplines including surgery, radiotherapy, chemotherapy, rehabilitation services, dental and prosthetic support, and psycho-social support are all crucial in bringing about a successful outcome of the therapeutic program. Management of cancer of the oral cavity is a multi-disciplinary team effort, and technical capabilities and support services from various disciplines are essential for a successful outcome $[3,7]$.

\section{Preoperative Assessment}

The preoperative assessment forms an important link between the diagnostic phase and treatment phase of a disease. This assessment includes clinical, radiographic, and histopathological assessment.

\section{The clinical assessment is dependent upon}

a) Site of the disease - For eg: Anterior lesions - transoral approach and posterior lesions- open resection.

b) Size of the disease- For eg: small lesions (t1) - transoral approach and large lesions ( $\mathrm{t} 2 / \mathrm{t} 3$ )-open resection.

The radiographical assessment includes CT/MRI to assess bone \& soft tissue invasion or PET-CT/ CT chest and ultrasound abdomen - to assess nodal involvement \& distant metastasis. Radiographic imaging is essential in the preoperative work-up to assess primary tumour extent, regional disease, and distant disease, and to identify synchronous second primary tumours. Evaluation of the head and neck most often involve highresolution anatomic imaging. This usually is done via computed tomography (CT) with intravenous contrast material because this imaging modality is accurate and relatively inexpensive for the assessment of bone invasion as well as soft tissue extent of the primary tumour and regional nodal disease. Magnetic resonance 
imaging (MRI) is superior for visualization of the soft tissue and hard palate and for evaluation of perineural invasion (PNI).

The histopathological assessment includes the use of intraoperative assessment/ frozen section to assess margin status. If the margins are at $>1 \mathrm{~cm}$ from the disease, then there is no need for re-resection. If the margins are $<1 \mathrm{~cm}$, then re-resection is done. The final histopathology report post-operatively helps to assess nodal involvement, extracapsular spread, perineural invasion and final staging. All these parameters form an important criterion for deciding the adjuvant therapy if any needed $[3,5,7]$.

\section{Approach to Surgical Resection}

The National Comprehensive Cancer Network (NCCN) recommends surgery for patients with early-stage tumours and surgery or definitive concurrent chemo radiotherapy for those with advanced-staged tumours. Surgery is often the first modality in sequential therapy because definitive high-dose radiation is associated with higher rates of Osteoradionecrosis. The goals of surgery are for complete resection of the primary tumour with negative margins and staging and treatment of regional lymphatics. Margin status is one of the most important variables associated with survival. There are various factors that affect the choice of a particular surgical approach for primary tumours of the oral cavity. These factors include the size of the primary tumour, its depth of infiltration, the site of the primary tumour (that is anterior versus posterior location) and proximity of the tumour to mandible or maxilla.

In addition to pre-operative clinical assessment of the primary tumour, examination under anaesthesia is often indicated to accurately delineate the extent of the tumour. The proximity of the tumour to the maxilla or mandible mandates the need for adequate clinical and radiographic assessment to rule out the possibility of bone invasion. The most employed imaging studies are a panoramic radiograph of the mandible (OrthopantomogramOPG) [5]. Besides these, a more detailed assessment of mandible invasion can be accomplished with a CT scan and a dental scan. On the other hand, magnetic resonance imaging provides a detailed assessment of the extent of tumour infiltration in the soft tissue.

The most employed surgical approaches for resection of primary oral cancer are peroral, mandibulotomy, lower cheek flap approach, visor flap approach or upper cheek flap approach. The visor flap avoids a lower lip splitting incision and provides satisfactory exposure only for the anterior aspect of the oral cavity. It, however, produces numbness of the skin of the chin due to the necessity to divide both mental nerves. Similarly, a sublabial degloving approach avoids an upper lip splitting Weber-Ferguson incision for resection of tumours of the anterior part of the nasal cavity and the infrastructure of the maxilla $[3,5]$.

Surgical Management of the Primary Tumor [3,7, 8-12]

\section{Tumours involving buccal mucosa [3, 8-9]}

The surgical approaches for primary tumours of buccal mucosa include following:

i. Peroral Approach- For accessible anterior lesions

ii. Cheek Flap

a. Midline lip split

b. Angle split (Algorithm 1 \& Figure 1)

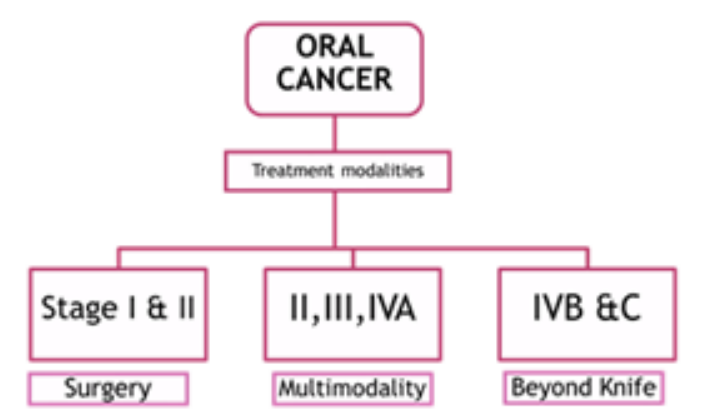

Algorithm 1: The various treatment modalities that can be used for tumors of buccal mucosa.

\section{Tumours involving tongue and its base $[3,8]$}

The surgical approaches for primary tumours of tongue mucosa include following:

i. Lesions in anterior tongue include

a. Transoral with electrocautery resection b. Transoral with median glossotomy (Algorithm 2)

If on CT assessment of bone - no bone invasion is seen, then the above two approaches without any bone sacrifice is enough. If on CT assessment of bone, it is found that bone is invaded then marginal/ segmental mandibulectomy is advised. 
ii. Lesions in posterior tongue/ with trismus/ obstructive dentition

a. Mandibulotomy with mandibular swing b. Suprahyoid approach

c. Lateral pharyngotomy with suprahyoid approach

d. Pull-through approach

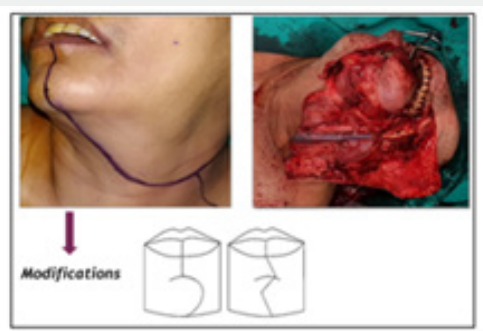

A

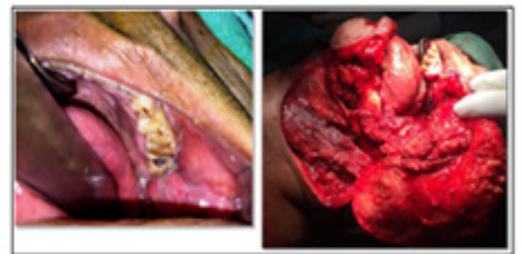

B

Figure 1: A- Midline lip split; B- Angle split.
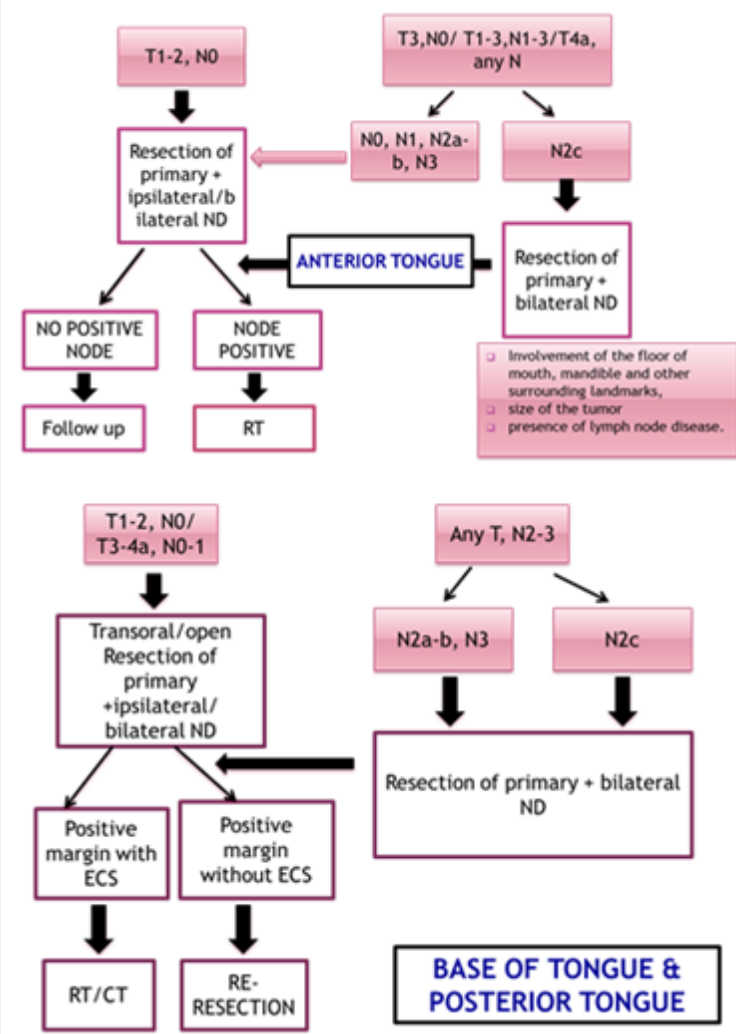

Algorithm 2: Treatment modalities for tumors involving tongue and base of tongue. 
If on CT assessment of bone - no bone invasion is seen, then the above two approaches without any bone sacrifice is enough. If on CT assessment of bone, it is found that bone is invaded then marginal/ segmental mandibulectomy is advised. Treatment strategy by $\mathrm{O}^{\prime}$ brien for tumours involving tongue includes

a) Initial surgery for primary cancer

b) Preservation of mandible whenever possible

c) Selective neck dissection for level 1-4 negative neck

d) Modified radical (or radical) neck dissection for clinically positive neck

e) Tracheostomy for advanced cancer

\section{Tumours involving floor of mouth $[3,10,11]$}

The surgical approaches for primary tumours involving floor of mouth include following: Lesion that are small ie T1/T2 lesions which are $1 \mathrm{~cm}$ away from mandible, Wide Local Excision (WLE) using the transoral approach is advised. The T3/T4 lesions i.e. lesions clinically larger than $2 \mathrm{~cm}$ in greatest dimension can be operated according to the bone involvement status (Algorithm 3). For lesions without involvement of mandible bone, the standard treatment is extended resection with/ without partial glossectomy via the Transcervical pull through approach. For T3/T4 lesions involving the mandible bone, extended resection with/ without partial glossectomy with/without segmental mandibulectomy is the recommended plan of resection using the Lower lip splitting incision and lower cheek flap (Algorithm 4).

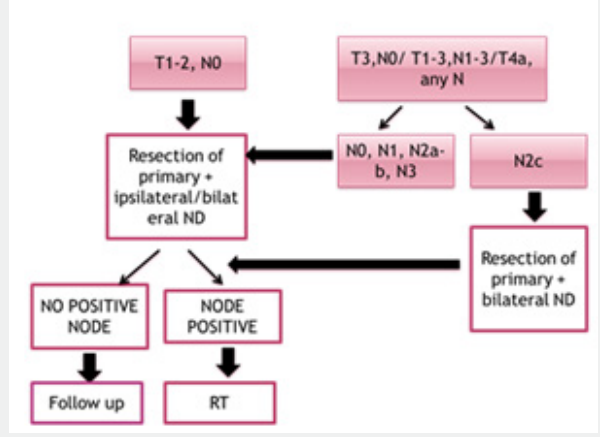

Algorithm 3: Treatment modalities for tumors involving floor of mouth.

\section{SURGICAL APPROACH}

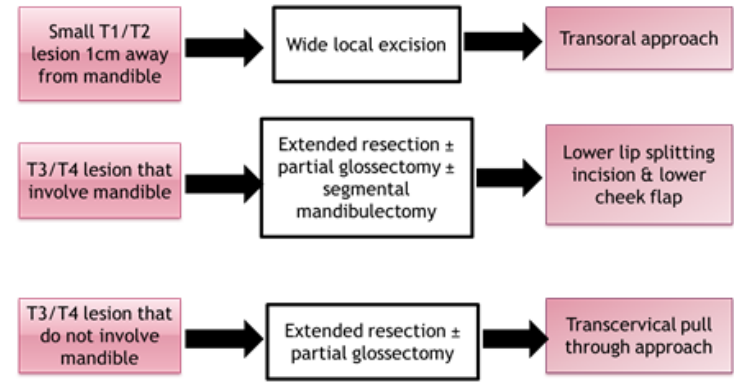

Algorithm 4: The surgical approaches for tumors involving floor of mouth.

\section{Tumour involving mandible}

The tumours involving the mandible bone can be classified as follows based upon the involvement of mandibular cortex by the tumour. T1 lesions show no bone invasion, T2 lesions are the ones with alveolar bone invasion. While the T3 andT4 lesions are distinguished based on the invasion in relation to the level of mandibular canal (Figure 2). The surgical approaches for tumours involving the mandible include following: i. Segmental resection/ mandibulectomy is advocated for tumours causing gross invasion of mandible along with Para mandibular soft tissue involvement via Lip splitting incision/ Lower cheek flap exposure/ Pull through approach.

ii. Marginal resection/ mandibulectomy is preferred for tumours of GB sulcus when mandible is in proximity to tumor but not involved or tumor of FOM abutting mandible. The approach used are Peroral approach/ Lip splitting incision (Figures 3 \& 4). 


\section{Global Journal of Otolaryngology}
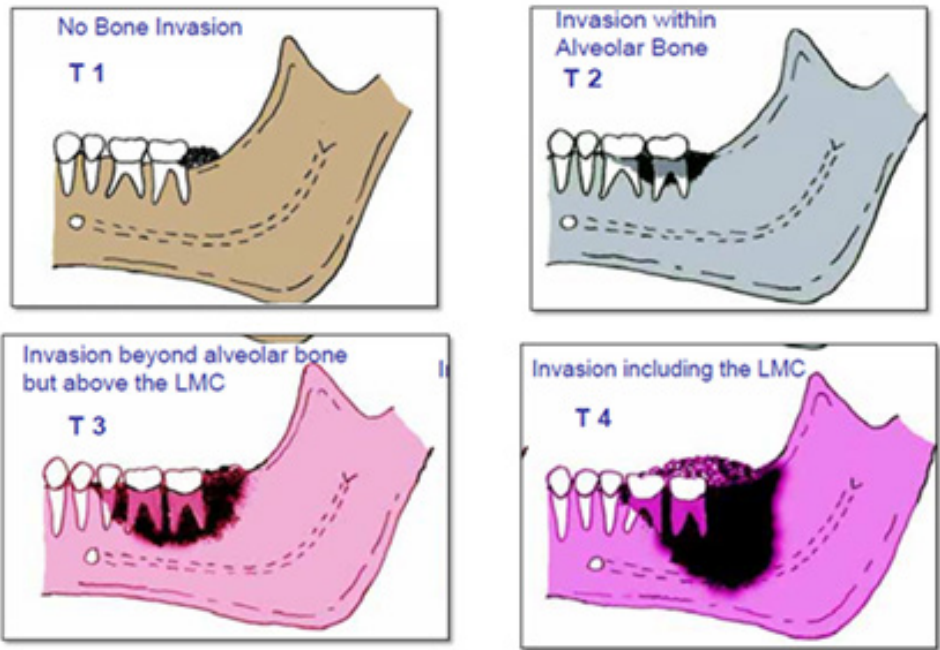

Figure 2: Pictorial representation of tumors involving mandible irt mandibular canal.

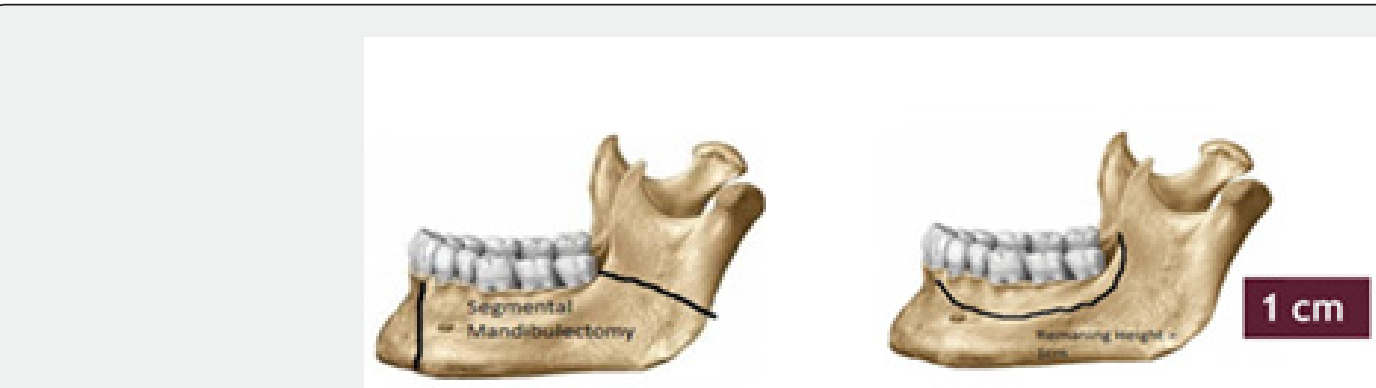

Figure 3: Pictorial representation for segmental and marginal mandibulectomy.
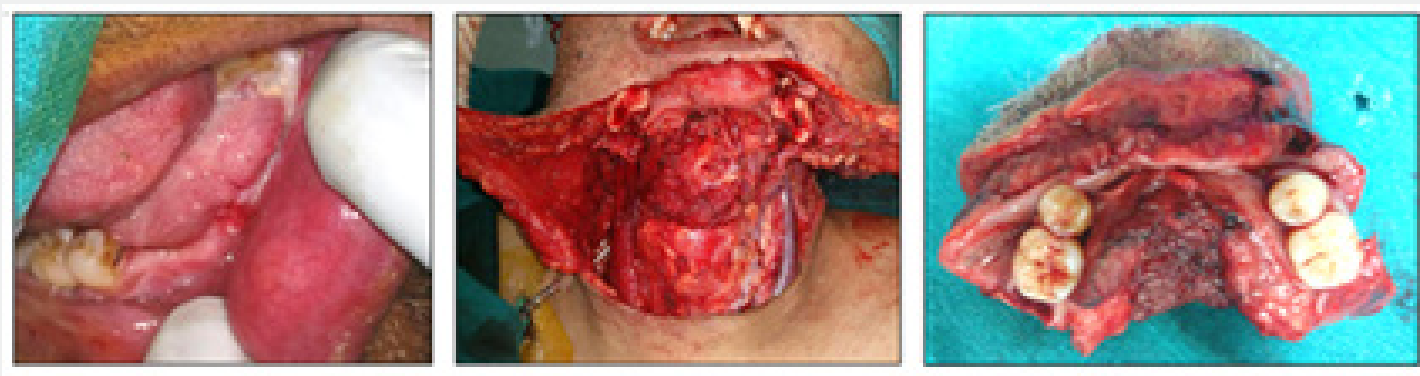

Figure 4: Intra-operative images of patient who underwent segmental mandibulectomy.

\section{Tumour involving maxilla $\&$ hard palate}

The most used approaches for tumours of maxilla and hard palate are:
a. Weber Ferguson
b. Midfacial degloving

For T1/T2 lesions, Wide local excision (WLE) with resection of involved mucoperiosteum \& bone is followed via the Transoral approach. For T3/T4 lesions with invasion of maxillary sinus/ nasal cavity - Partial maxillectomy through the Weber Ferguson/ midfacial degloving approach. Advanced lesions invading pterygomaxillary space and pterygoid plate undergo Subtotal Maxillectomy via the Weber Ferguson method (Figures 5 \& 6). 

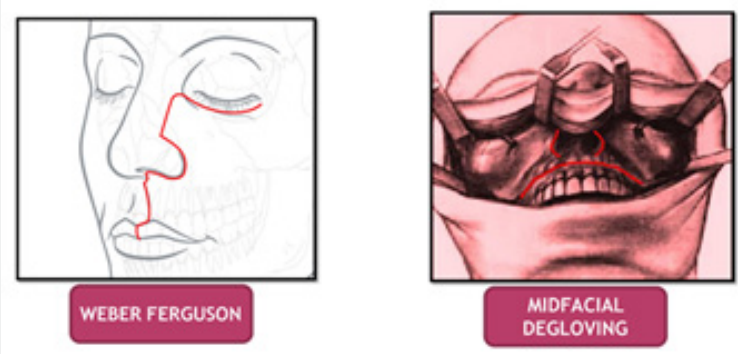

Figure 5: Diagrammatic representation of surgical approaches for tumours of maxilla and hard palate.
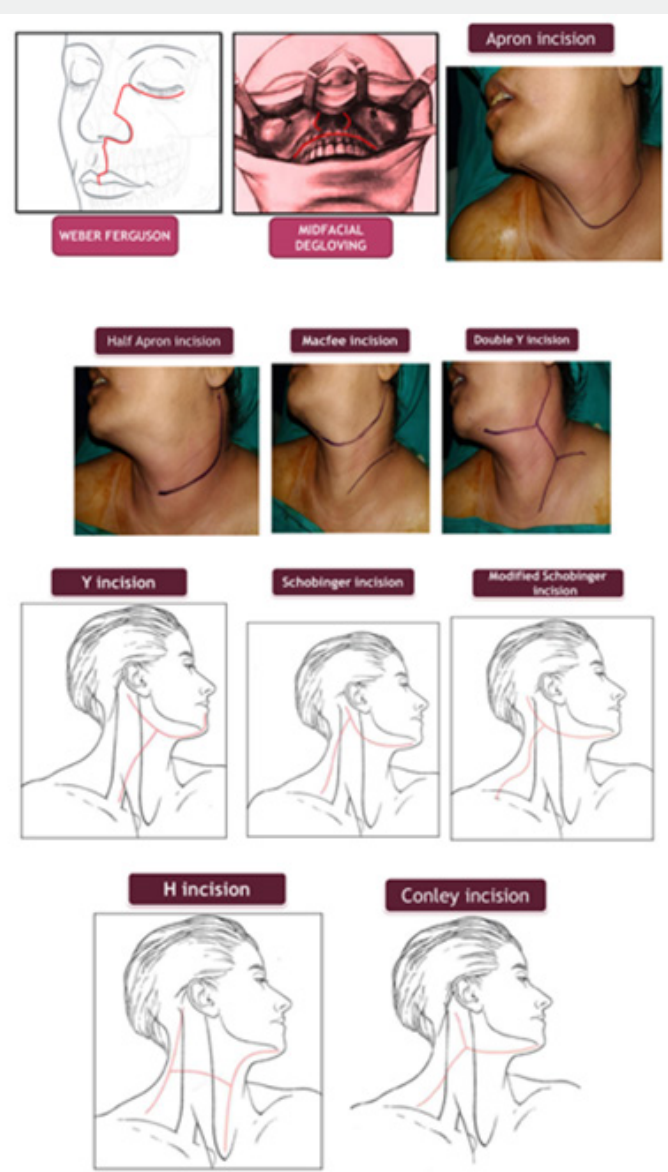

Figure 6: Representation of various surgical incisions.

\section{Management of Neck $[3,13]$}

Neck dissection is the surgical procedure in which the fibrofatty contents of the neck are removed for the treatment of cervical lymphatic metastases (Figure 7).

a. Remove gross disease in patients with clinical evidence of nodal involvement (therapeutic neck dissection)

b. Remove occult metastases in patients whose tumor characteristics make one suspicious of occult cervical metastases (elective neck dissection or END).

The Memorial Sloan-Kettering Cancer Center in the 1930s developed a system to classify the cervical lymph nodes, the system divides the lymph nodes in the lateral aspect of the neck into five nodal levels, I through $\mathrm{V}$. The lymph nodes in the central compartment are in category level VI, and those in the superior anterior mediastinum are level VII. The clinical and surgical landmarks for neck node levels are: 




Figure 7: Diagrammatic and post-operative (before grossing) picture of neck dissection.

A. Level I (submental and submandibular triangles) borders are:

a. Superior - the lower border of the body of the mandible.

b. Posterior - posterior belly of digastric

c. Inferior - hyoid bone

B. Level II (upper jugular lymph nodes) borders are:

a. Superior - the base of the skull

b. Posterior - posterior border of the sternocleidomastoid

c. Anterior - lateral limit of sternohyoid

d. Inferior-hyoid bone

C. Level III (middle jugular lymph nodes) borders are:

a. Superior - hyoid bone

b. Posterior - posterior border of the sternocleidomastoid

c. Anterior - lateral limit of sternohyoid

d. Inferior - cricothyroid membrane

D. Level IV (lower jugular lymph nodes) borders are:

a. Superior - cricothyroid membrane

b. Posterior - posterior border of the sternocleidomastoid

c. Anterior - lateral limit of sternohyoid

d. Inferior - clavicle

E. Level V (posterior triangle of the neck) borders are:

a. Posterior - the anterior border of the trapezius

b. Anterior-theposteriorborder of thesternocleidomastoid

c. Inferior - clavicle
F. Level VI: (central compartment of the neck) borders are:

a. Superior - hyoid bone

b. Inferior - suprasternal notch

c. Lateral - medial border of the carotid sheath on either side

G. Level VII: (superior mediastinal lymph nodes) borders are:

a. Superior - suprasternal notch

b. Inferior - innominate artery

Neck dissection can be classified according to the structures and levels of cervical lymph nodes that are sacrificed.

\section{Neck Dissection (ND)}
A. Comprehensive ND
a. Radical ND
b. Modified radical ND
B. Selective ND
a. Supraomohyoid ND
b. Lateral ND
c. Anterolateral ND
C. Extended ND (Algorithm 5)

\section{Radical neck dissection}

Lymph nodes from level I-V, ipsilateral sternocleidomastoid muscle (SCM), internal jugular vein (IJV), and spinal accessory nerve (SAN) undergo removal. RND is indicated when $\mathrm{N}+$ the neck for squamous cell carcinoma (SCC) where SAN involved and/ or extensive soft tissue disease with the invasion of SCM and IJV (Figure 8). 




Figure 8: Pictorial representation of Radical Neck Dissection.

\section{Modified radical neck dissection}

Excision of same lymph node bearing regions as RND with preservation of one or more non- lymphatic structures (SAN, SCM, IJV) (Figure 9).

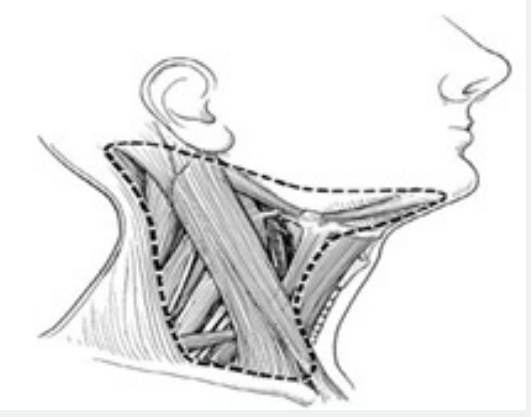

Figure 9: Pictorial representation of Modified Radical Neck Dissection.

a. Modified radical neck dissection type I (MRND-I): Lymph nodes from level I-V, ipsilateral sternocleidomastoid muscle, and internal jugular vein get removed, with preservation of the spinal accessory nerve. Indications for MRND-I is in N+ the neck for SCC where SAN is free of disease.

b. Modified radical neck dissection type II (MRNDII): Removal of lymph nodes from level I-V and ipsilateral sternocleidomastoid muscle, with preservation of IJV and SAN. The N+ neck for SCC or thyroid cancer where IJV involved but SAN is free of disease.

c. Modified radical neck dissection type II (MRND-III): Lymph nodes from level I-V undergo removal, with preservation of SCM, IJV, and SCM. MRND-III is indicated in metastatic differentiated thyroid carcinoma (Figure 10).

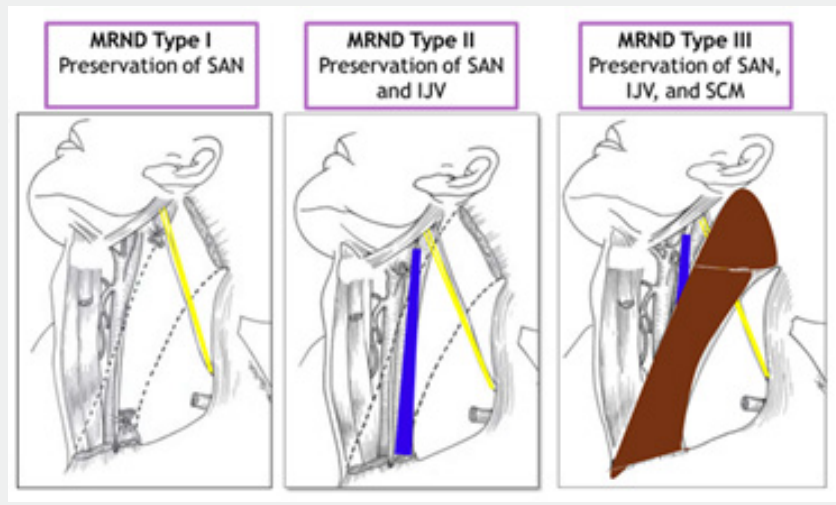

Figure 10: Pictorial representation of types of Modified Radical Neck Dissection. 


\section{Global Journal of Otolaryngology}

The severe comorbidities related to the removal of SCM, IJV, and SAN led to the numerous modifications of the procedure with selective removal of lymph nodes. They are:

i. $\quad$ Supraomohyoid neck dissection (SOHD): Lymph nodes removed are Levels I-III, with sparing of IJV, SCM, and SAN. SOHD is indicated in N0 neck for SCC of oral cavity and oropharynx (include level 4) and N0 neck malignant melanoma where primary site is anterior to ear (include parotidectomy for face and scalp).

ii. Extended supraomohyoid neck dissection (Ex SOHD): Removal of lymph nodes in levels I-IV, with sparing of IJV, SCM, and SAN. The procedure is indicated in N0 neck for SCC of the lateral tongue. iii. Lateral neck dissection: Removal of lymph nodes from level II-IV with sparing of IJV, SCM, and SAN. Indications for this procedure are in N0 neck for SCC of larynx and hypopharynx.

iv. Postero-lateral neck dissection: Removal of levels II-V, suboccipital, retro-auricular nodes with sparing of IJV, SCM, and SAN. Indications for this procedure is in N0 neck malignant melanoma where the primary site is posterior to ear.

v. Modified lateral neck dissection: Removal of levels II to V lymph nodes with relative sparing of SCM, IJV, SAN, and submaxillary gland. This procedure has an indication of therapeutic neck dissection for thyroid cancer with lateral neck nodes (Table 1).

Table 1: Patterns of neck nodal metastasis.

\begin{tabular}{|c|c|}
\hline Primary Site & First Echelon Nodes \\
\hline Oral cavity & Levels I, II, III \\
\hline Larynx, Pharynx & Levels II, III, IV \\
\hline Thyroid & Levels IV, VI, superior mediastinal \\
\hline Parotid & Levels II, III, Pre-auricular, Peri \& intra parotid, Upper accessory chain \\
\hline Submandibular, sublingual glands & Level I, II, III \\
\hline
\end{tabular}

\section{Mendenhall's criteria}

Algorithm for treating an N0 (Algorithm 6 \& Table 2).

Table 2: Mendenhall's Criteria.

\begin{tabular}{|c|c|c|c|c|}
\hline Group & $\begin{array}{c}\text { Estimated Risk of Subclinical } \\
\text { Neck Disease }\end{array}$ & T stage & Site & Treatment \\
\hline Low risk I & $<20 \%$ & $\mathrm{~T} 1$ & FOM, Tongue, Gingiva, Hard palate, BM, RMT & Under observation \\
\hline \multirow{2}{*}{$\begin{array}{l}\text { Intermediate } \\
\text { risk II }\end{array}$} & \multirow{2}{*}{$20-30 \%$} & $\mathrm{~T} 1$ & Soft palate & \multirow{2}{*}{ Neck dissection may be required } \\
\hline & & $\mathrm{T} 2$ & FOM, Tongue, Gingiva, Hard palate, BM, RMT & \\
\hline \multirow{3}{*}{ High risk III } & \multirow{3}{*}{$>30 \%$} & T1-T4 & BOT & \multirow{3}{*}{ Neck dissection mandatory } \\
\hline & & $\mathrm{T} 2-\mathrm{T} 4$ & Soft palate & \\
\hline & & T3-T4 & FOM, Tongue, Gingiva, Hard palate, BM, RMT & \\
\hline
\end{tabular}

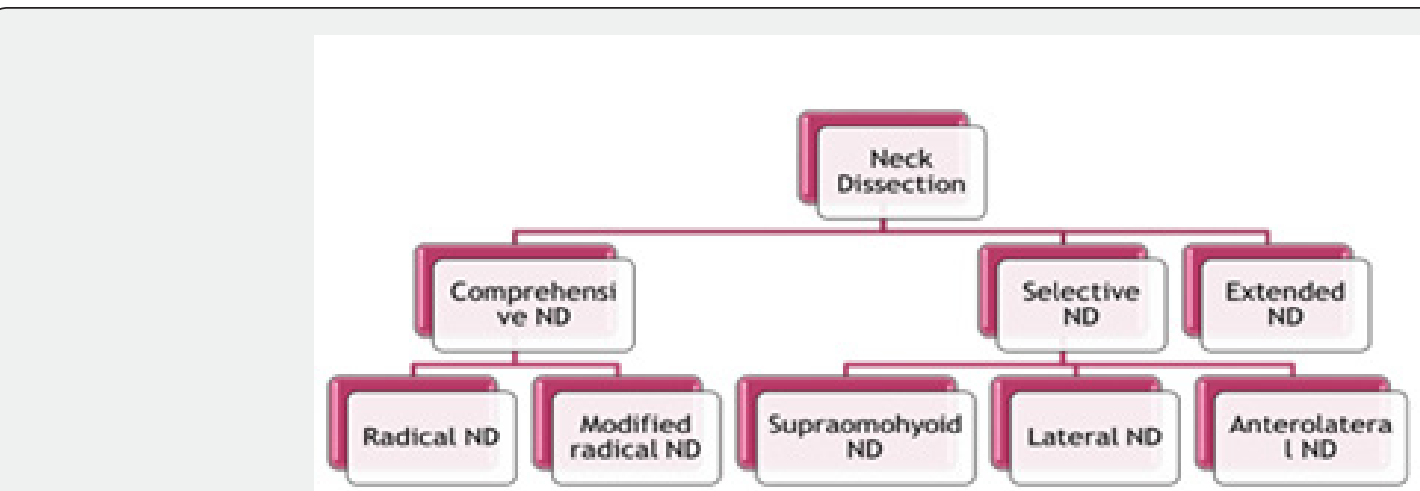

Algorithm 5: Classification of Neck Dissection. 


\section{$\mathrm{N}+$ disease needs comprehensive neck dissection}

a. SCC anterior to circumvallate papilla Supraomohyoid Neck Dissection (SOHND)

b. SCC Oropharynx, larynx and hypopharynx Level I- IV or level II-V neck dissection

c. SCC with N+ nodes Radical Neck Dissection (TND)

d. SCC with 2-4 positive nodes or extracapsular spread Radical Neck Dissection (RND) and adjuvant therapy.

\section{Conclusion}

Thus, to conclude from this article we can understand that the entire process of decision making involves multiple steps and is directly/indirectly affected by many factors. These factors can be patient dependent or clinician dependent or disease dependent. The final treatment protocol decided for each patient is customized according to each individual and cannot be standardized for every patient. Therefore, a in depth understanding of the procedures and awareness about the latest techniques or advances in treatment modalities is a mandate for each professional.

\section{References}

1. Rawat G, Urs AB, Chakravarti A, Kumar P (2018) Evaluation of DNA Damage in Peripheral Blood Leukocytes in Oral Potentially Malignant and Malignant Disorders by Comet Assay. Clin Cancer Investig J 7: 5055.

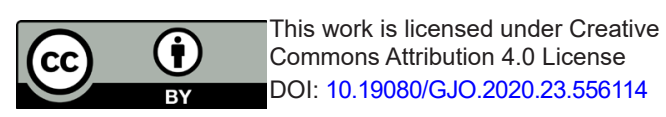

2. Chinn SB, Myers JN (2015) Oral Cavity Carcinoma: Current Management, Controversies, and Future Directions. J Clin Oncol 33(29): 3269-3276.

3. National Comprehensive Cancer Network Guidelines.

4. Shah JP, Patel SG (2003) Head and Neck Surgery and Oncology. (3 $3^{\text {rd }}$ edn), Edinburgh, London, New York.

5. Werning JW, Byers RM, Novas MA, Roberts D (2001) Preoperative assessment for and outcomes of mandibular conservation surgery. Head Neck 23(12): 1024-1030.

6. Shah JP (1990) Patterns of cervical lymph node metastasis from squamous carcinomas of the upper aerodigestive tract. Am J Surg 160(4): 405-409.

7. Shah JP, Gill Z (2009) Current concepts in management of oral cancer surgery. Oral Oncol 45(0): 394-401.

8. Shah JP (2007) Surgical approaches to the oral cavity primary and neck. Int J Radiat Oncol Biol Phys 69(2): S15-S18.

9. Chaukar D, Dandekar M Surgical resection of cancer of the buccal mucosa. Open access atlas of otolaryngology, head \& neck operative surgery.

10. Fagan J (2017) Resection of cancers of the base of tongue. Open access atlas of otolaryngology, head \& neck operative surgery 1 .

11. Fagan J (2017) Resection of floor of mouth cancer. Open access atlas of otolaryngology, head \& neck operative surgery 1.

12. Shah J P, Johnson N W, Batsakis J G (2003) Oral Cancer. London: Martin Dunitz, pp. 387-394.

13. Harish K (2005) Neck dissections: radical to conservative. World J Surg Oncol 3(1): 21.

\section{Your next submission with Juniper Publishers will reach you the below assets}

- Quality Editorial service

- Swift Peer Review

- Reprints availability

- E-prints Service

- Manuscript Podcast for convenient understanding

- Global attainment for your research

- Manuscript accessibility in different formats

( Pdf, E-pub, Full Text, Audio)

- Unceasing customer service

Track the below URL for one-step submission https://juniperpublishers.com/online-submission.php 